\title{
Cavity and Interface effect of PI-Film on Charge Accumulation and PD Activity under Bipolar Pulse Voltage
}

\author{
Shakeel Akram*, Guangning Wu*, GuoQiang Gao ${ }^{\dagger}$, and Yang Liu*
}

\begin{abstract}
With the continuous development in insulation of electrical equipment design, the reliability of the system has been enhanced. However, in the manufacturing process and during operation under continues stresses introduce local defects, such as voids between interfaces that can responsible to occurrence of partial discharge (PD), electric field distortion and accumulation of charges. These defects may lead to localize corrosion and material degradation of insulation system, and a serious threat to the equipment. A model of three layers of PI film with air gap is presented to understand the influence of interface and voids on exploitation conditions such as strong electrical field, PD activity and charge movement. The analytical analysis, and experimental results are good agreement and show that the lose contact between interfaces accumulate more residual charges and in consequences increase the electric field intensity and accelerates internal discharges. These residual charges are trapped charges, injected by the electrodes has often same polarity, so the electric field in cavities increases significantly and thus partial discharge inception voltage (PDIV) decreases. Contrary, number of PD discharge quantity increases due to interface. Interfacial polarization effect has opposite impact on electric field and PDIV as compare to void.
\end{abstract}

Keywords: Partial discharge, Surface charge, Bipolar square pulse waveform, Polyimide film, Breakdown, Electric field distortion

\section{Introduction}

Development, in electronics has made three-phase AC motors an alternative to the DC motor because improvements in power converter technology allow pulsewidth modulated (PWM) inverter to drive electric motors on adjustable speed in industry $[1,2]$. On the other hand such high speed repetitive square pulse supplied by PWM can damage insulating material. Polyimide film as an insulating material had been used in many areas such as microelectronics, electric industries, motor winding and aerospace [3]. Since field distortion, partial discharge and space charge due to interface and void defects lead to early failure and material aging. Internal partial discharges between interface occur is recognized as the main cause of change in electric field. This electric field distortion causes breakdown accident, influence the safety of the normal operation of the motor.

To improve the insulation reliabilities of inverter-fed motors, the International Electrotechnical Committee (IEC) released technical specifications (TS), prescribing how PD detections should be performed on new insulation systems

$\dagger$ Corresponding Author: School of Electrical Engineering Southwest Jiaotong University Chengdu, 610031, China (xnjdggq@home. swjtu.edu.cn, shakil uetians2005@yahoo.com)

* School of Electrical Engineering Southwest Jiaotong University Chengdu, 610031, China

** School of Electrical Engineering and Information Sichuan University Chengdu, China.

Received: September 22, 2014; Accepted: May 24, 2015 or new motor stator windings $[4,5]$. When evaluating the harmful effects caused by PD on inverter-fed motor insulation systems based on the TS, lifetime tests should be implemented on both the insulation models and the stator windings to evaluate the corona resistance capability of motor insulation system although a small amount PD is allowed when it is in service [5]. Therefore, the PDIV should be detected as an acceptance test to verify that this value is high enough to ensure that PD will not occur during the expected lifetime of the motor [4].

In fact extensive studies have been done and are going on, such as injection of charges inside dielectric materials under high electric field can be known through the electronic structure at interface [6], interfacial defects or traps. Consecutive conduction of charges inside dielectrics is due to the band conduction or defect states inside dielectrics $[7,8]$.

Day by day components size shrinking to nano scale, the chemical and physical structure of surfaces at interface within the dielectric has gained a lot of attention and can sometimes dominate other properties. So, it is also important to study PI structure to understand the charge injection and its movement between interfaces.

This paper covers the following main topics

- Electric field enhancement and PD activity in the aged sample having internal voids.

- Interface polarization and cavity discharge effect on $\mathrm{PD}$ and voltage endurance. 
- Interface effect on electric breakdown and injected charge mobility.

Analytical, Experimental study is put forward to solve above issues. The results show that the electric field distortion, depend on both electrode arrangement as well as the defect within the insulation material. The permittivities of insulation material are higher than air, and in some conditions high electric field can occur in localized region where the permittivities change abruptly. Mostly it arises at intersecting point of two or more materials with different permittivity like layer/layer or layer/air/layer and especially more strong when intersecting points are non orthogonal. The strongest field mostly generate in the material of lowest permittivity. All the tests are perform on repetition square voltage waveform because PD mechanism for pulse voltage is different than sinusoidal waveform due to fast front edge rise and high frequency [9] and the memory effect of residual charges in voids under bipolar square waveform is deeper than sinusoidal waveform [10].

\section{Analytical Analysis of Model}

\subsection{Interface Effect on Electric field with and without Charge Accumulation Inside Material}

To understand the relation of charge (residual charge, space charge) influence on electric field distortion and partial discharge inception voltage in presence of cavity, an analytical model is presented. The air gap between layers is considered as cavity defect in this model. Fig. 2 shows on left side air gap defected turn winding and in (A) layer/air/layer insulation system and (B) accumulation of charge density inside layer I because in the presence of strong electric field charges can accumulate anywhere between interfaces or within one layer due to local defects. Possible partial discharge location inside stator winding of inverter fed motor is shown in Fig. 1.

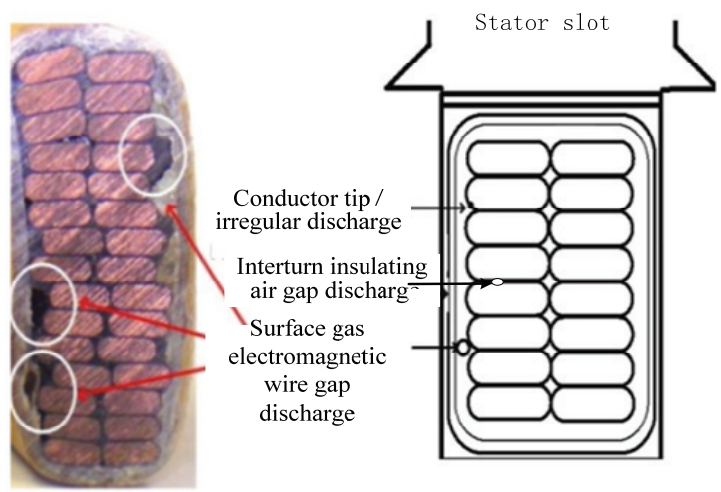

Fig. 1. Partial discharge location in high pressure molding motor stator

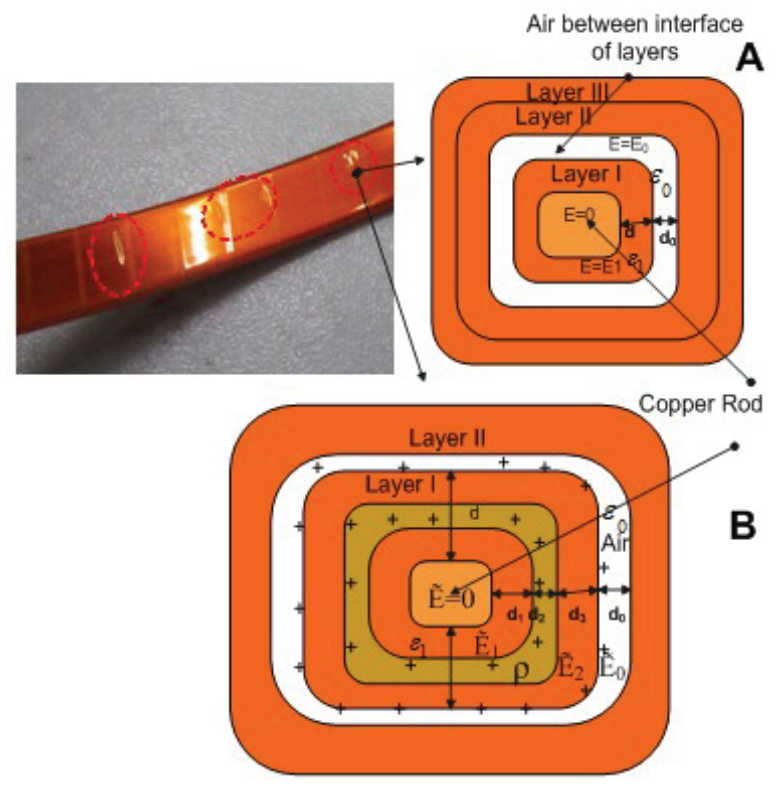

Fig. 2. Electric field behavior (A) Without any charge accumulation inside layer (B) With space charge and interface charge accumulation inside layer and on layer-air interface (Only half part consider due to symmetry)

To reduce complexity and better understanding only two layers are considered and bulk of charges present inside layer I as shown in Fig. 2 (B) because the insulation system is symmetrical and layer I is zoomed. In Fig. 2 (A), no space charge and no interface charge.

Assume sample is symmetrical so, with no space charges accumulation inside material as well as in air/insulation interfaces Fig. 2 (A), the air cavity electric field $E_{0}$ is given as

$$
\begin{gathered}
U=q / c \text { and } E=U / d \\
E_{0}=\frac{U}{\frac{\varepsilon_{1}}{\varepsilon_{0}} d+d_{0}}
\end{gathered}
$$

Where $\varepsilon_{1}$ and $\varepsilon_{0}$ are the dielectric constant of polyimide film layer I and air respectively, $U$ is the voltage, $d$ is the layer insulation thickness and $d_{0}$ is the cavity thickness. As $\varepsilon_{1}>\varepsilon_{0}$ and $E_{0}=\frac{\varepsilon_{1}}{\varepsilon_{0}} E_{1}$, so $E_{0}>E_{1}$ and the space charge accumulation in internal and external interference can be derived using Eq. (2) [11, 12]. There can be two reasons for charges accumulation, one is the charges injection from electrode inside material other can be cavity surface PD activity [11]. Consider $\rho$ is the volume charge density inside layer I as shown in (figure B), so total thickness of layer $\mathrm{I}$ is $d_{1}+d_{2}+\mathrm{d}_{3}=d$ and charge per unit surface at interface is $\sigma \mathrm{s}$, in the presence of volume charge density the electric field change to $\tilde{E}$, and can be given using Maxwell's equations. 


$$
\left\{\begin{array}{c}
E=-\nabla U \\
\nabla^{2} U=-\rho / \varepsilon \text { When bulk of charges present } \\
\nabla \cdot \tilde{E}=0 \text { When no charges present }
\end{array}\right\}
$$

Let suppose the thickness $d_{2}$ of the charge distribution $(\rho)$ is very small as compared to the insulation thickness $d$, the relation of $\tilde{E}_{1}$ and $\tilde{E}_{2}$ as shown in Fig. B can be given as Eq. (4).

$$
\tilde{E}_{1}=\tilde{E}_{2}+\frac{\rho d_{2}}{\varepsilon_{1}}
$$

After introducing charge distribution inside layer the $\tilde{E}_{0}$ inside cavity depend on the interference $\left(\sigma_{s}\right)$ charges and can be given as equation

$$
\tilde{E}_{0}=\frac{\varepsilon_{1}}{\varepsilon_{0}} \tilde{E}_{1}+\frac{\sigma_{s}}{\varepsilon_{0}}
$$

Due to symmetry

$$
\tilde{E}_{1} d_{1}+\tilde{E}_{2} d_{3}+\tilde{E}_{0} d_{0}=U
$$

From Eq. (4) and (5) get value of $\tilde{E}_{1}$ and $\tilde{E}_{2}$ and expressed $\tilde{E}_{1}$ and $\tilde{E}_{2}$ in terms of $\tilde{E}_{0}$ then substitute into Eq. (6) and get Eq. (7).

$$
\tilde{E}_{0}=\frac{U}{\frac{\varepsilon_{0}}{\varepsilon_{1}} d_{1}+\frac{\varepsilon_{0}}{\varepsilon_{1}} d_{3}+d_{0}}+\frac{\frac{\sigma_{s}}{\varepsilon_{1}} d_{1}+\frac{\sigma_{s}}{\varepsilon_{1}} d_{3}+\frac{\rho d_{2}}{\varepsilon_{1}} d_{3}}{\frac{\varepsilon_{0}}{\varepsilon_{1}} d_{1}+\frac{\varepsilon_{0}}{\varepsilon_{1}} d_{3}+d_{0}}
$$

Since $d \tilde{=} d_{1}+d_{3}$ (if $d_{2}<<d_{1}$ and $d_{2}<<d_{3}$ )

$$
\tilde{E}_{0}=\frac{U}{\frac{\varepsilon_{0}}{\varepsilon_{1}} d+d_{0}}+\frac{\frac{\sigma_{s}}{\varepsilon_{1}} d+\frac{\rho d_{2}}{\varepsilon_{1}} d_{3}}{\frac{\varepsilon_{0}}{\varepsilon_{1}} d+d_{0}}
$$

The first part of Eq. (8) is the electric field in air mean ( $\rho$ and $\sigma_{s}=0$ ) denoted as $E_{0}$ from Eq. (2), so final expression is

$$
\tilde{E}_{0}=E_{0}+\frac{\sigma_{s} d+\rho d_{2} d_{3}}{\varepsilon_{0} d+\varepsilon_{1} d_{0}}
$$

It can be observed that in case of homocharges that mean if $\rho$ and $\sigma_{s}$ has the similar polarity like electrode near which they accumulated, the electric field $\tilde{E}_{0}$ enhanced as compared to $E_{0}$ as shown in Eq. (9). In case of heterocharges the electric field decreases.

\subsection{Interface Effect on Electric Field and Charge Density with Only Surface Charge Present on Layer}

Suppose $\rho=0$, only charges present on the interference surface Eq. (9) become as

$$
\tilde{E}_{0}=E_{0}+\frac{\sigma_{s} d}{\varepsilon_{0} d+\varepsilon_{1} d_{0}}
$$

The values of $\sigma_{s}$ depend on the traps at interfaces; these traps appear only in case of DC component or at low frequency of PWM supply voltage.

We indicate $\kappa_{0}, \kappa_{1}$ are the electrical conductivities of air and insulation layer respectively, while, $\varepsilon_{0}, \varepsilon_{1}$ are dielectric constants of air and insulation layer respectively, $D_{0}$ and $D_{l}$ are flux densities of air and insulation layer respectively, $J_{0}$ and $J_{l}$ are current densities of air and layer insulation respectively. For discontinuance surfaces it can be written from Maxwell Eqs. [13].

$$
\begin{aligned}
& \tilde{D}_{0}-\tilde{D}_{1}=\sigma_{s} \\
& \tilde{J}_{0}-\tilde{J}_{1}=\frac{\partial \sigma_{s}}{\partial t}
\end{aligned}
$$

Since $D=\varepsilon E$ and $J=\kappa E$

$$
\begin{gathered}
\varepsilon_{0} \tilde{E}_{0}-\varepsilon_{1} \tilde{E}_{1}=\sigma_{s} \\
\kappa_{0} \tilde{E}_{0}-\kappa_{1} \tilde{E}_{1}=-\frac{\partial \sigma_{s}}{\partial t}
\end{gathered}
$$

Interface charge density can be obtained from Eq. (13) and (14) and given as

$$
\sigma_{s}=\tilde{E}_{0}\left(\varepsilon_{0}-\varepsilon_{1} \frac{\kappa_{0}}{\kappa_{1}}\right)
$$

Under interface charge the electric field notation can be obtained by substituting Eq. (15) into Eq. (10) and obtain Eq. (16).

$$
\tilde{E}_{0}=\frac{E_{0}}{1-\frac{\varepsilon_{0}-\varepsilon_{1} \frac{\kappa_{0}}{\kappa_{1}}}{\varepsilon_{0}+\varepsilon_{1} \frac{d_{0}}{d}}}
$$

According to above equation it can be seen that, $\varepsilon_{1}>\varepsilon_{0}$ and $\kappa_{0} \gg \kappa_{1}$ after PD occurs inside the cavity then $\left(\varepsilon_{0}-\varepsilon_{1} \frac{\kappa_{0}}{\kappa_{1}}\right)<0, \sigma_{s}$ sign is opposite to $\tilde{E}_{0}$. Therefore presence of interface charges may decrease the electric field inside cavity and partial discharge inception voltage may increase.

\section{Experimental Analysis}

\subsection{Sample}

The inter-turn insulating sample having capacitance of 


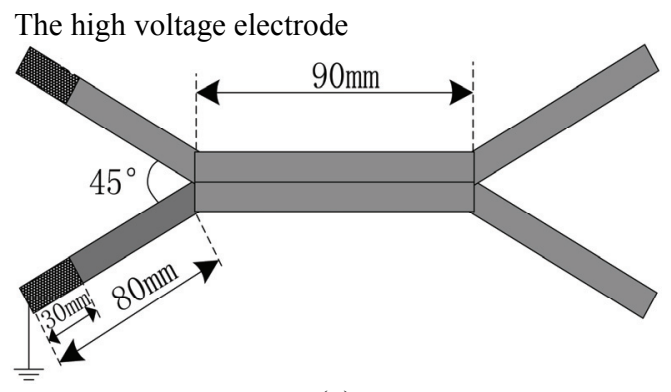

(a)

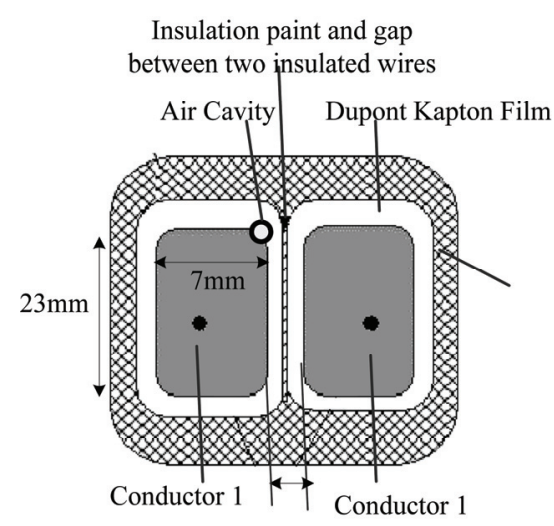

(b)

Fig. 3. Configuration of an insulating magnet wires: (a) front view of sample; (b) sectional view of sample

about $37.4 \mathrm{pF}$ is shown in Figure 3. These insulating wires are folded using DuPont KAPTON 100CR film that is polyimide film with the thickness of $25 \mu \mathrm{m}$. After overlapping two wires with PI film lapped once with glass ribbon. One of the specimens is vacuum pressure impregnated (VPI) to reduce the micro bubbles in the interface. The middle length of wires is $90 \mathrm{~mm}$, the curving part is $80 \mathrm{~mm}$, and the intersection angle is 45 degree. The cross-sectional view details are shown in Fig. 3(b). The samples are dried for four hours at $110^{\circ} \mathrm{C}$ temperature and then cool in air for $30 \mathrm{~min}$. before testing. VPI and without applying VPI samples are used to measure PD discharge quantity and amplitude.

\subsection{Test voltage}

The PWM square wave bipolar pulse voltage with variable repetition frequency is used in inverter-fed traction motor. IEC 62068-1 [14] suggests that bipolar pulse voltage can replace PWM voltage supply for testing. All tests are studied at peak to peak voltage magnitude of 3500 $\mathrm{V}$, frequency $1 \mathrm{kHz}$, rise time $1 \mu \mathrm{s}$ and duty cycle $50 \%$.

\subsection{PD measurement}

PD measuring apparatus is shown in Fig. 4. High Voltage HV power supply developed by the research group was adopted. It can supply maximum peak-to-peak value of pulse voltage is up to $7 \mathrm{kV}$, duty cycle is $50 \%$, and frequency is from $50 \mathrm{~Hz}$ to $10 \mathrm{kHz}$, rise time is $100 \mathrm{~ns}$ or more. PD signals are transferred by high frequency current transformer (HFCT), which is made of coils and high frequency magnetic core of nickel-zinc composite material. A non-inductive resistor of $50 \Omega$ is used to match the impedance between HFCT sensor and oscilloscope. To avoid the distortion of high frequency PD signals, the bandwidth of HFCT is chosen from $5 \mathrm{MHz}$ to $500 \mathrm{MHz}$, a high-pass filter with $40 \mathrm{MHz}$ was placed between the resistor and oscilloscope, which is used to obtained original signal and restrain interference signal caused by power supply impulses. PD impulse spectrum is always broader [15]. To obtain PRPD pattern under bipolar square pulse voltage, high voltage probe is utilized. PD signals are measured constantly under sampling rate of $500 \mathrm{MHz}$ for 15 times during each cycle of bipolar impulse voltage.

Due to high frequency square wave pulse voltage with very steep rising edge (high $\mathrm{dV} / \mathrm{dt}$ ), partial discharge signal

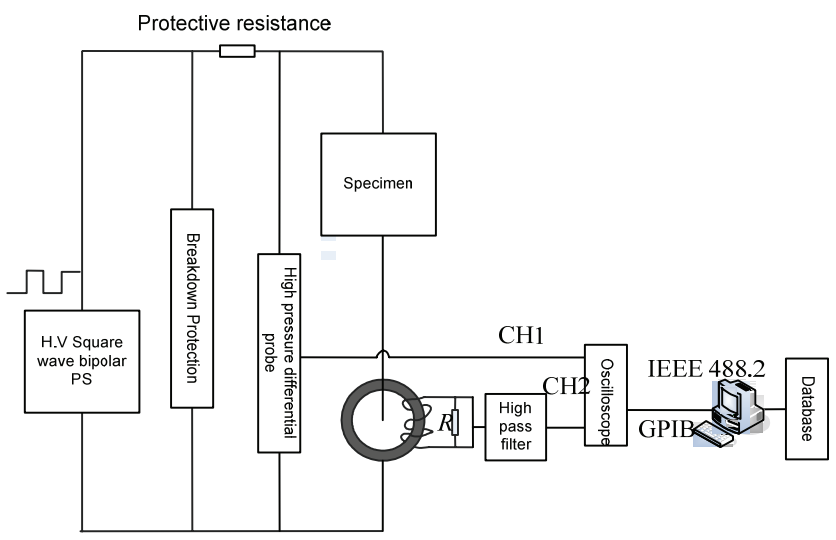

Fig. 4. Schematic diagram of PD testing under bipolar pulse voltage

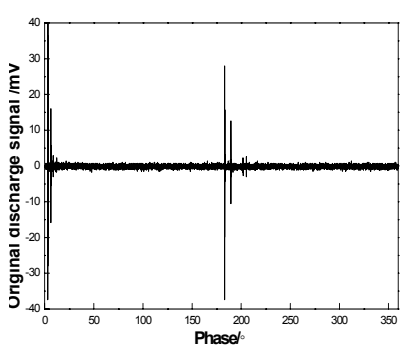

(a) The original signal

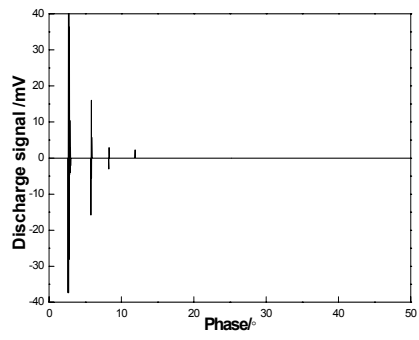

(c) Remove interference

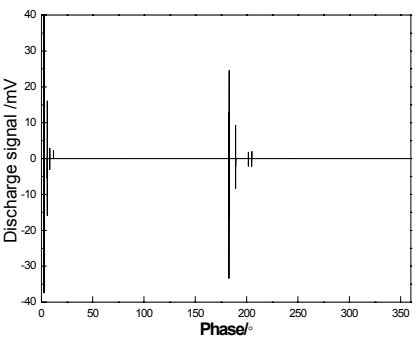

(b) Signal threshold filtering

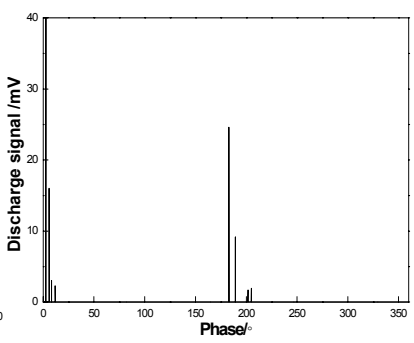

(d) Partial discharge signal
Fig. 5. Partial discharge signal extraction process 
contains interfering signal component, for the accurate analysis of PD, it needs to deal with the original partial discharge signal, The dB6 wavelet packets method can effectively separate low frequency and high frequency segments, so it is used to get PD signal from interference signal as shown in Fig. 5.

\section{Results of Partial Discharge}

Before measuring PRPD pattern PD inception voltage (PDIV) and PD extinction voltage (PDEV) is measured. At a frequency of $1 \mathrm{kHz}$ and rise time of $1 \mu \mathrm{s}$, for several specimens, PDIV is in the range of 2200-3000 V (voltage peak-to-peak $\left(\mathrm{V}_{\mathrm{pp}}\right)$ and PDEV is in the range of 2200-2600 $\mathrm{V}\left(\mathrm{V}_{\mathrm{pp}}\right)$ at room temperature while other temperature effect on PDIV is shown in Fig. 6. Before measuring PRPD pattern samples are heated again for 30 minutes at $250{ }^{\circ} \mathrm{C}$.

PRPD patterns are shown in Fig. 7 and Fig. 8, the

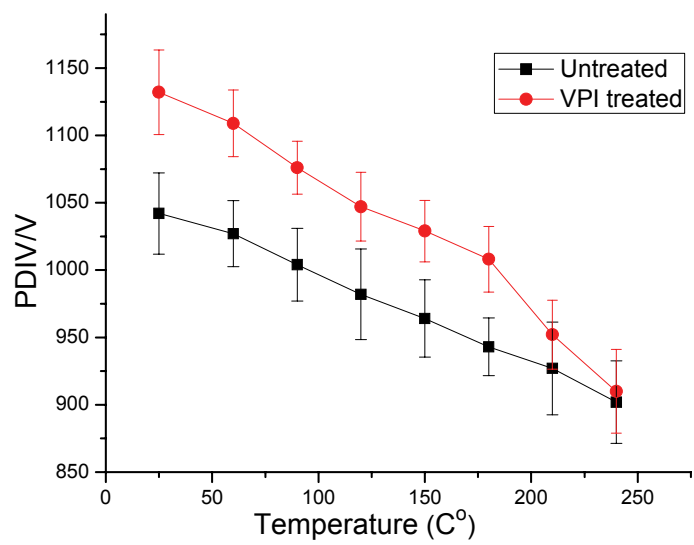

Fig. 6. Effect of temperature on PDIV
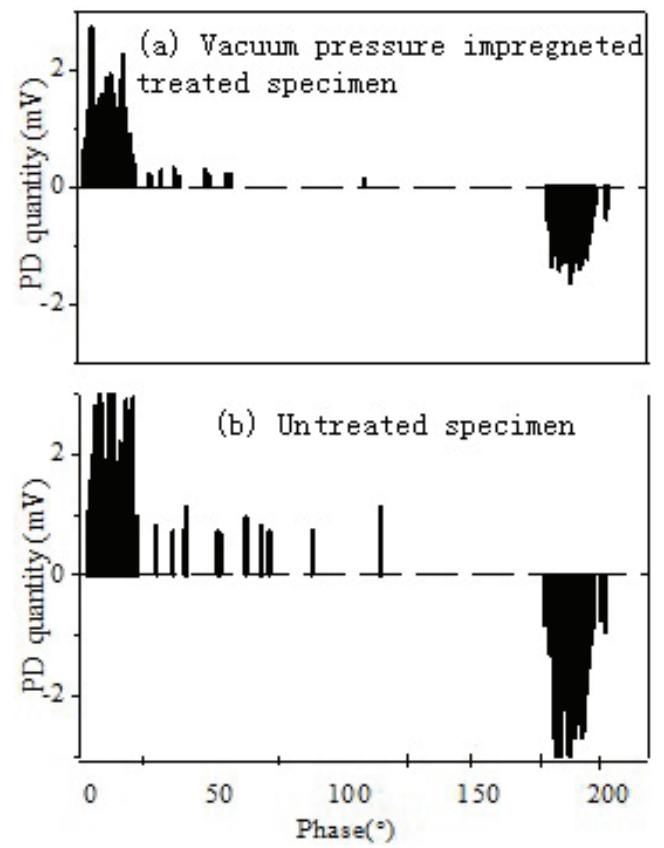

Fig. 7. PD quantities with respect to phase distribution.
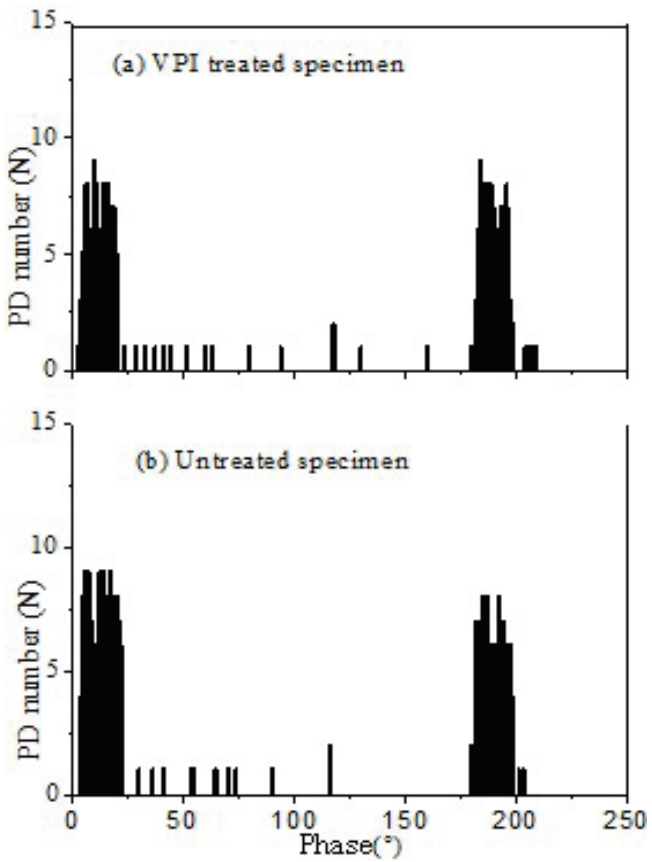

Fig. 8. Relevant phase distribution of PD number

amplitude and discharge quantity is up to several nano coulombs (nC). It is observed that most of PDs occur at rise and fall slopes of a bipolar pulse cycle, very few PDs also present at flattop of bipolar pulse waveform. Amplitude of discharge quantity and discharge number is almost symmetric between positive and negative half-cycle as shown in Fig. 7 and Fig. 8 respectively. Therefore, it can be predicted that PDs of insulating wires under bipolar pulse is mostly due to internal discharges (cavity and interface).

PD only occurs if two conditions are satisfied: one is the applied voltage reaches or exceeds PDIV; the second is to have enough free electrons to form electron avalanche discharge. The dissipation of heat between interfaces and cavities are difficult, so temperature play important role to increase kinetic energy of molecules. Elevated temperature increases thermal excitation of trap charges, increases the probability of obtaining the initial electrons for discharge, reduces the required discharge time, and at the same time reduces PDIV. High temperature increases electrical activity and random motion of gas molecules and to make it easier for ionization, Therefore, PDIV reduce at high temperature and partial discharge activity can aggravate as shown in Fig. 6. PD amplitude and number is higher in untreated specimens due to high electric field intensity near cavity and availability of enough free electrons for avalanche and discharge. The reason of this is discussed below in discussion part.

\subsection{SEM image}

Fig. 9 shows the microscopic view of dissembled PI film after PD and it tell us that most of the discharge occurs 

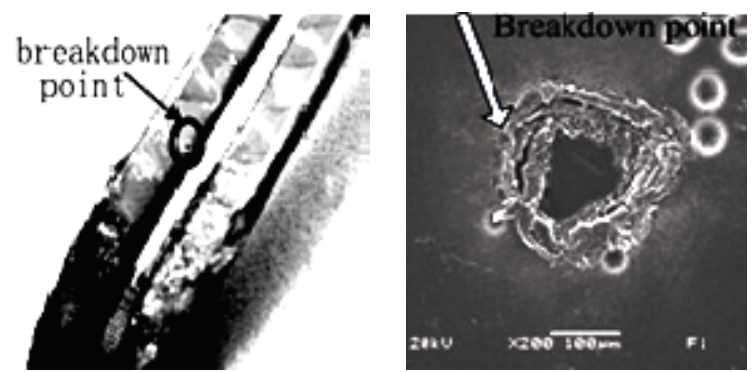

Fig. 9. Disassembled twisted pair wires and magnifying images of turn insulation

around cavity and interface between layers. Most discharge appears around the air bubble gap and other cavities between two wires. The surface around this area is eroded as the result of PD discharge that promotes to the roughness and structure change.

Therefore electric field intensity increases in this area causes breakdown. These voids within the layer are due to material deterioration. Due to structural change and physical morphology change the cavity size can be increased. The chances of increasing cavity sizes are high in multilayer due to the degradation caused by cavity discharge. If these cavities are supplied constantly to a higher voltage than PDIV, insulating material degradation due to discharge will occur, and failure is certain.

\section{Discussion}

According to the above results it is discussed that how pattern of PD belong to internal discharge inside voids and interfaces between electrode/layer and layer/Air/layer.

\subsection{PD activity and influence of space charge inside air gap cavity}

A lot of work has already been done by many authors to describe PD activity models for single void that can explain their experimental results under different voltage waveforms and frequencies $[16,17]$. In order to understand PD activity inside air gap, it is necessary to analyze a complete PD process during a bipolar pulse cycle. According to the above results and SEM image most of the PD pattern relates to internal discharge in the air gap cavity. A complete PD process and change in electric field on the basis of experimental results are shown in Figure 10 and detail is described below.

Assuming that $E(t)$ with the maximum magnitude $E$ is externally applied electric field to the cavity, $E_{P}$ is internal electric field generated by space charges in the cavity. Total electric field $E_{i}$ of the cavity could be obtained by difference between $E$ and $E_{P}$. In Fig. 10(a), when starting electron is available and supply voltage cross the limit of PDIV of the cavity, a discharge would occur. Afterwards, total electric field $E_{i}$ of the cavity would decrease to

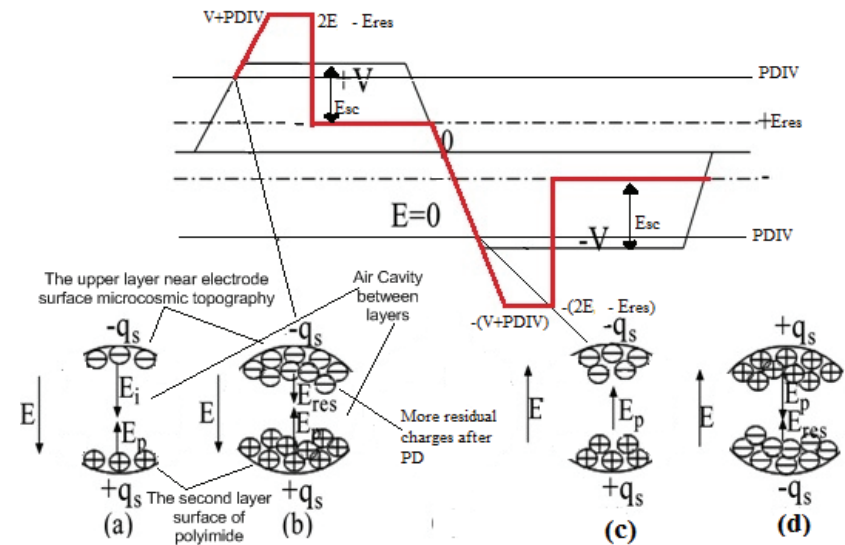

Fig. 10. Change of electric field inside cavity during a bipolar pulse cycle.

residual value $E_{\text {res. }}$. If the charge injection rate is faster than the charge depletion rate then the field due to space charge will deposit and remain on the insulation surface i.e., $\mathrm{E}_{\mathrm{sc}}$. Since more residual charges generated by previous discharge in the cavity enhance $E_{P}, \mathrm{E}-\mathrm{E}_{\mathrm{p}}$ could not reach minimum breakdown electric field $E_{\min }$ and PD is extinguished, as shown in Fig. 10(b). Polarity of bipolar impulse voltage changes very quickly, so polarity of residual space charges in the cavity could not change immediately and only few charges could vanish in time. As a result, magnitude and polarity of $E_{P}$ approximately keep invariant. When external electric field $E$ changes the polarity, the total field around cavity equals to $\left(E+E_{P}\right)$, shown in Fig. 10(c). Because electric filed $E_{P}$ governed by space charges in the cavity nearly equals $\left(E-E_{\text {res }}\right)$, the total electric field in cavity could be represented as $(2 E-$ $\left.E_{\text {res }}\right)$. Then another discharge starts in the cavity. Total electric field in the cavity drops to $E_{\text {res }}$ again and space charges in the cavity move to opposite position due to the effect of electric field, as shown in Fig. 10(d). Because $E_{P}$ generated by residual charges after previous discharge is enhanced, $\left(E-E_{P}\right)$ could not reach $E_{\min }$ and $\mathrm{PD}$ is extinguished again. Consequently, a PD process during a whole bipolar impulse cycle is completed. According to the changes of electric field in the cavity in Fig. 10, variation of the corresponding voltage across the cavity under a bipolar pulse cycle could be obtained. The maximum magnitude of voltage $\left(V+V_{P D I V}\right)$ across the cavity would appear at the rise slope and fall slope of bipolar impulse. At flattop of bipolar impulse, because discharge has started in the cavity at the rise slope and fall slope, the voltage across the cavity would drop to residual voltage $V_{\text {res }}$ and few discharges would occur.

\subsection{PD activity and influence of space charge between interfaces}

In order to explain the interface effect on charge injection, charge transport and recombination, a model is presented 


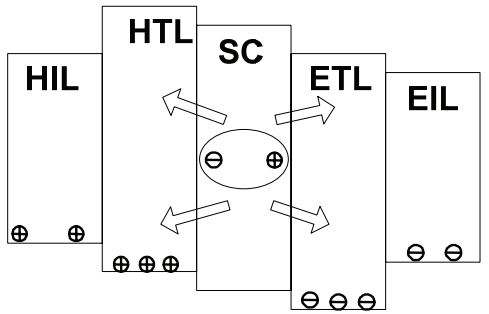

Fig. 11. Multilayer structure, hole injected layer (HIL), hole transfer layer (HTL), electron transfer layer (ETL), electron injected layer (EIL)

on the basis of above results as shown in Fig. 11. It also helps to understand interface effect on electric field distortion, PD process and space charge. Suppose our sample consist of two thin layers sandwiched by two electrodes. One layer near positive electrode is considered for holes transportation and other near cathode for electrons transportation. These layers interfaces provide sufficient area for recombination of injected holes and electrons. If the electric field at anode is at higher voltage than cathode. Holes from HIL enter into (HTL) and injected electrons from EIL to ETL as shown in Fig. 11.

These charge carriers move towards opposite charge electrodes and some recombine near the interface junction. The junction between HTL and ETL represent space charge region. These residual charges are already present due to previous PD effect. This SC region can act as hetro or homo charge junction. In case of homo charge junction it can promote holes from HTL to ETL and stop electrons in the reverse direction to increase the probability of recombination near interface region. In case of hetro charge junction the opposite happened. HTL is little above than ETL, so that holes could easily enter into the ETL. The low mobility of holes in ETL accelerate or build hole density, and lead collision. In addition, if the interface contact spacing is large then the chances of suppressing near the electrode area is much reduced.

When charges are injected from the electrodes, it is necessary that charges should cross potential barriers and make bridge at interfaces of electrode and insulation. These hurdles depend on the electrode material type and corona resistance of insulation surface. According to the Mott Schottky model [6] the Fermi level of electrodes and insulating material are repeated over the interface, lead to three type of contacts: ohmic, neutral, and blocking. Let $\Psi_{\mathrm{m}}$ and $\Psi_{\mathrm{s}}$ are the work functions of electrode material and insulating material respectively then if $\Psi_{\mathrm{m}}=\Psi_{\mathrm{s}}$ it is neutral. In this case no charges transfer across the materials and conduction band remains empty at interface. If $\Psi_{\mathrm{m}}>\Psi_{\mathrm{s}}$, it is ohmic. In this case electrodes can provide charges to overcome for those available at insulating material. Negative space charge (SC) region is formed when electrode inject electrons towards conduction band of material. This type of contact can supply many electrons to the material. When $\Psi_{\mathrm{m}}<\Psi_{\mathrm{s}}$ it block electrons movement from the conduction band of material to the electrode forming negative $\mathrm{SC}$ region. The low densities of electrons in this area make most of the voltage drop across this contact.

\section{Results of Breakdown}

Weibull statistical probability method is used to compare the measured breakdown strength. Glass transition temperatures (Tgs) usually exceed $300^{\circ} \mathrm{C}$ for rigid ordered polyimides, but those of $400{ }^{\circ} \mathrm{C}$ or above are preferred for interlayer dielectrics [18]. In this experiment temperature range between $25^{\circ} \mathrm{C}$ to $400{ }^{\circ} \mathrm{C}$ is selected. The results in Fig. 12 and Fig. 13 show the polyimide film breakdown mechanism mainly for electric and thermal breakdown process. PI molecular chain containing six benzene ring and five imide ring structure, molecular structure, has strong limitations on the molecular chain, the PI material has strong heat resistance. However, with the increase of temperature, the breakdown field strength reduced. In this temperature range, with the increase of temperature, the intensity of electric field under the same conditions,

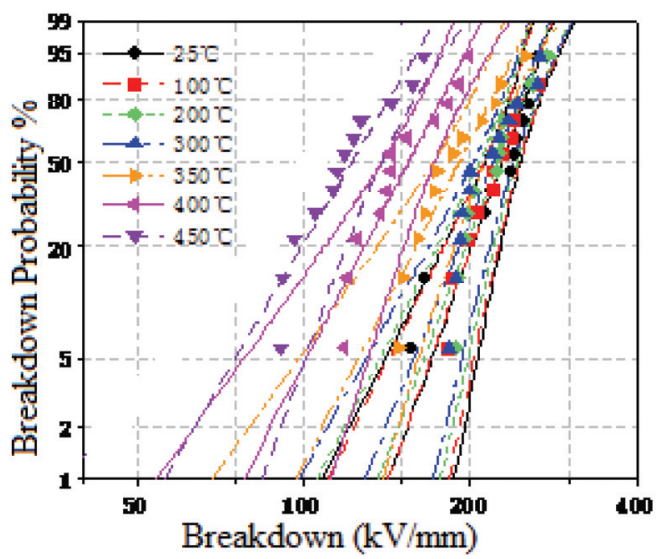

(a)

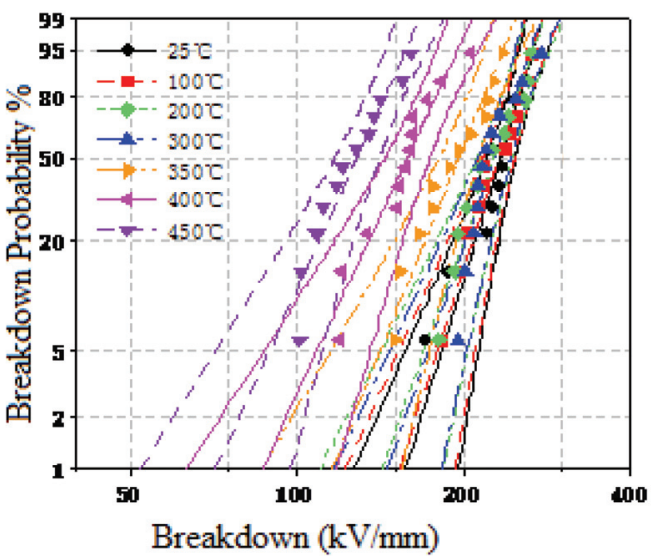

(b)

Fig. 12. Weibull statistic of Breakdown strength: (a) untreated specimen; (b) VPI treated specimen 


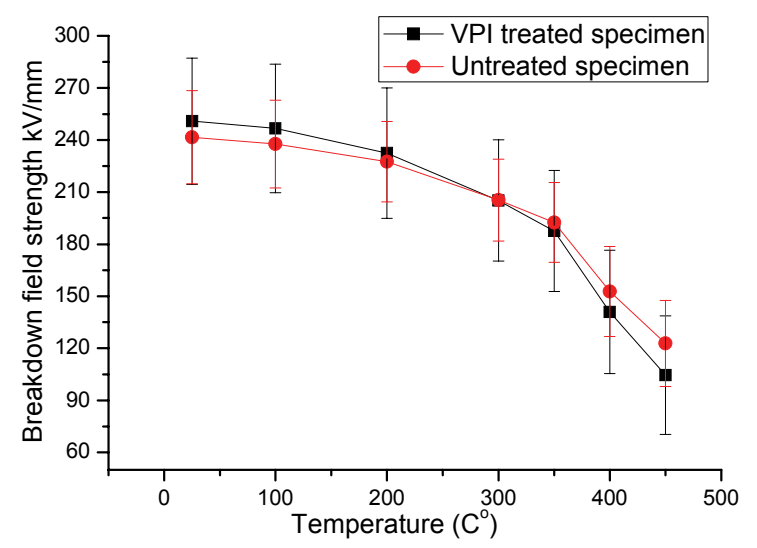

Fig. 13. Effect of cavity and interface on the breakdown strength of specimens with increase in temperature

enhance the ability of the electron emission, the number of charges injected into PI film increases, while heat provide internal excitation and make electrons deep trap into the conduction band.

Thermal effects can also force the residual chemical group and impurities and due to thermal imidization process internal un-reacted ions are released, this increases the number of carriers inside the material. High temperature reduce the interaction between molecular chain and chain segments tend to be more liberal pattern movement, light trap quantity increase helps to enhance the migration of charge carriers; finally, the heat will cause the PI medium microspore and free volume increases, so that the electron process continues to accelerate energy charge, and higher in the presence of strong external electric field. Therefore, with the increase of test temperature increased the number of carrier; enhance the transportation capacity of the charge carrier, kinetic energy collision increases, at the same time dielectric strength gradually decreased with the increase of temperature.

In order to know the interface effect breakdown tests for single, two and three layers are measured under different temperature using rod type electrode specifications of this electrode given in our previous paper [19]. Three layers of

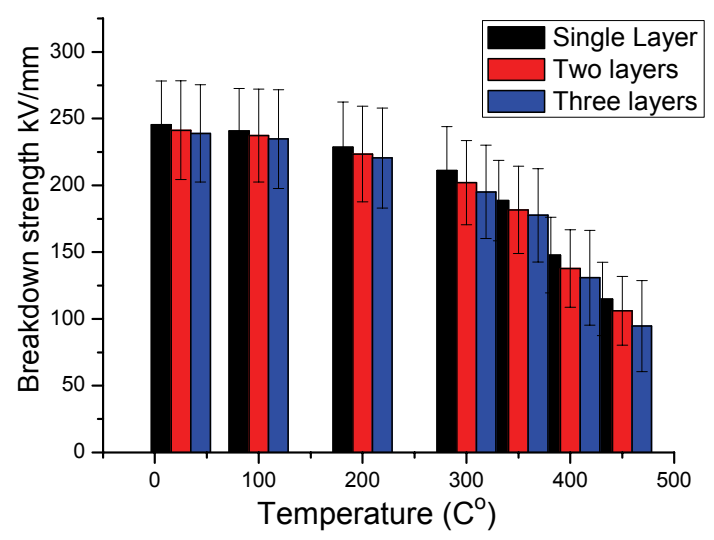

Fig. 14. Breakdown strength of layers at different temperatures
PI films overlapped together to form a composite insulation structure. Thermal conductivities of such structure are low and heat cannot dissipate easily. Multilayer film interface having micro cavities and other insulation defects increase the number of inhomogeneity and weakens the insulation structure. It can gather a large number of space charges in the interface trap inside. This will cause the local stress distortion, reduce the breakdown field strength. With the increase of temperature, the insulation defects at the interface further enlarged and breakdown strength reduced as shown in Fig. 14 [20].

\section{Conclusion}

In this work the factors like interface and formation of voids after electric stresses is considered and it is tried to explain that how these factors can affect the life of insulation material especially used in inverter fed induction motors. The main factors of PI film deterioration are the cracking of polymer chains and cavities. Mostly, physical morphology change and chemical deterioration are caused by discharge (e.g., PD) and accumulation of charges (e.g., surface charge, space charge). Discharges generate following process as listed below.

- High energy electrons responsible of electric field enhancement around cavities and ultraviolet rays, during discharge, affect the polymer chain.

- Temperatures can go high due to discharge that melts the local zone of the polymer.

- Material degradation due to electric stresses alters the chemical structure and changes the crystallites of material and form cavities and interface within the material.

If cavity discharge occurs, and heat due to high temperature could not dissipate immediately then the above mentioned effects concentrate on the interface layer of PI film and breakdown is certain.

\section{Acknowledgements}

The research presented in this paper was supported by the National Key Basic Research and Development Plans (Grant NO. 2011CB711100), Natural Science Foundation of China (51107104), (U1234202). The authors would like to thanks the reviewers for their valuable comments that helped us to improve the quality of the paper.

\section{References}

[1] W. Yin, "Dielectric properties of an improved magnet wire for inverter-fed motors," IEEE Elect. Insul. Mag., 
Vol. 13, No. 4, pp. 17-23, 1997.

[2] S.U. Haq, S.H. Jayaram, and E.A. Cherney, "Insulation problems in medium voltage stator coils under fast repetitive voltage pulses," IEEE Trans. Industry Appl., Vol. 44, No. 4, pp. 1004-1012, 2008.

[3] L. Mascia and A. Kioul, "Influence of siloxane composition and morphology on properties of polyimidesilica hybrids", Polymer, vol. 36, no. 19, pp. 36493659, 1995.

[4] IEC 60034-18-41: Rotating electrical machines - Part 18-41: Qualification and type tests for Type I electrical insulation systems used in rotating electrical machines fed from voltage converters, 2006.

[5] IEC TS 60034-18-42: Rotating electrical machines Part 18-42: Qualification and acceptance tests for partial discharge resistant electrical insulation systems (Type II) used in rotating electrical machines fed from voltage converters, 2008.

[6] J. Robertson, O. Sharia, A. Demkov, “A Fermi level pinning by defects in $\mathrm{HfO}_{2}$-metal gate stacks", Appl. Phys. Lett. 91:132912, 2007.

[7] G. Teyssedre, C. Laurent, "Charge transport modeling in insulating polymers: from molecular to macroscopic scale", IEEE Trans. Dielect. Electr. Insul. Vol. 12, No. 5, pp. 857-875, 2005.

[8] D. Arnold, E. Cartier, D. J. DiMaria "Theory of highfield electron transport and impact ionization in silicon dioxide", Phys Rev B Vol. 49, pp. 10278-10297, 1994.

[9] L. Tong, G. Wu, and L. Xi, "Relationship of partial discharge and insulation life," International Conference on Condition Monitoring and Diagnosis, pp. 662-665, Changwon, Korea, 2-5 April 2006.

[10] Cavallini, R. Ciani, M. Conti, P. F. H. Morshuis, and G. C. Montanari, "Modeling memory phenomena for partial discharge processes in insulation cavities," Annual Report Conference on Electrical Insulation and Dielectric Phenomena, pp. 723-727, 2003.

[11] D. Fabiani, Accelerated degradation of ac-motor winding insulation due to voltage waveforms generated by adjustable speed drives, Ph.D. Thesis, University of Bologna, Bologna, Italy, 2002, published by Gedit Edizioni, Bologna, Italy, May 2003.

[12] M. Takashima, K. Sda and T. Takada, "Measurement of electric charges at the interface between two dielectric layers using an electro-acoustic transducer technique", IEEE Trans. Electr. Insul., Vol. 23, pp. 287_295, 1988.

[13] T. Jing, Surfac charge accumulation in SF6, Ph.D. Thesis, Delft University Press, Delft, The Netherlands, 1993.

[14] IEC 62068-1. Evaluation of electrical stresses produced b repetitive impulses. Part 1: General methods of evaluation of electrical endurance. IEC 62068-1, 2003.

[15] IEC 61934. Electrical insulating materials and systems - Electrical measurements of partial discharges during short rise time repetitive voltage impulses. IEC 61934, 2006.

[16] D. Fabiani, G. C. Montanari, and A.Contin, "Aging acceleration of insulating materials for electrical machine windings supplied by PWM in the presence and in the absence of partial discharges," IEEE ICSD, pp. 283-286, Eindhoven, The Netherlands, 25-29 June 2001.

[17] R. Bodega, P. H. F. Morshuis, M. Lazzaroni, and F. J. Wester, "PD recurrence in cavities at different energizing methods," IEEE Trans. Instrumentat. Measur., Vol. 53, No. 2, pp. 251-258, 2004.

[18] K. Horie, T. Yamashita, Photosensitive Polyimides: Fundamentals and Applications; Technomic: 1995.

[19] Luo Yang, Wu Guangning, Wang Peng, Zhang Yiqiang, Cao Kaijiang, Xu Huihui, Xin Zhengliang, "Effect of Temperature on Partial Discharge Properties of Polyimide Film Under Continuous Square Impulse Voltage", Proceedings of the CSEE Vol.32 No.19, pp. 154-160, Jul.5, 2012.

[20] http://dx.doi.org/10.1080/15325008.2013.816979.

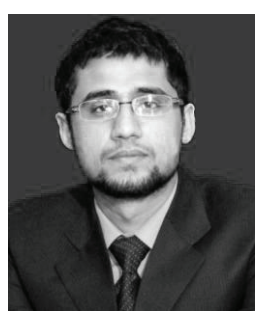

Shakeel Akram was born in Punjab, Pakistan in 1987. He received the B.Sc. degree in electrical engineering from the Bahauddin Zakariya University Multan, Pakistan. He received his M.Sc. degree in electrical engineering from Chongqing University Chongqing, China. Currently he is pursuing his Ph.D. degree in Electrical Engineering from Southwest Jiaotong University Chengdu, China. His research interest in High Voltage Technology is Space Charge, Partial Discharge, Breakdown testing and material degradation mechanism under square wave pulse voltage.

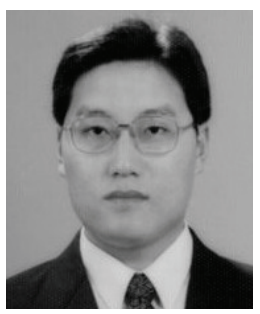

G. N. Wu (M'97) was born in Nanjing, China in July 1969. He received the B.Sc., M.Sc. and Ph.D. degrees in electrical engineering from Xi'an Jiaotong University, respectively in 1991, 1994 and 1997. Currently, he is Professor in the Scholl of Electrical Engineering, Southwest Jiaotong University. His research interests include condition monitoring, fault diagnosis and insulation life-span evaluation for electric power equipment. 


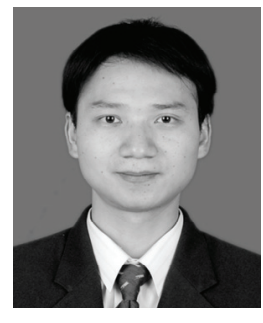

Guo Qiang Gao was born in Hubei province, China on 22 October 1981. $\mathrm{He}$ received the B.Sc. and M.Sc. degrees in physics in 2003 and 2006, respectively and the Ph.D. degree in electrical engineering in 2012 from Southwest Jiaotong University Chengdu, China. Currently, he is an Associate Professor in the School of Electrical Engineering, Southwest Jiaotong University and his research interests are High Speed Railway technology and high voltage technology.

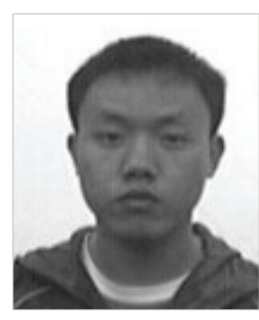

Liu Yang was born in Guangyuan, China, in June 1991. He received the B.Sc. degree in electrical engineering from Southwest Jiaotong University China, in 2009. He is now pursuing the M.Sc. degree in the School of electrical Engineering from SWJTU Chengdu China. Currently, his research interests are in failure mechanism for electrical insulation. 